\title{
Diagnosis and Treatment Protocol for Novel Coronavirus Pneumonia
}

\author{
(Trial Version 7) \\ (Released by National Health Commission \& State Administration of \\ Traditional Chinese Medicine on March 3, 2020)
}

Since December 2019, multiple cases of novel coronavirus pneumonia (NCP) have been identified in Wuhan, Hubei. With the spread of the epidemic, such cases have also been found in other parts of China and other countries. As an acute respiratory infectious disease, NCP has been included in Class B infectious diseases prescribed in the Law of the People's Republic of China on Prevention and Treatment of Infectious Diseases, and managed as an infectious disease of Class A. By taking a series of preventive control and medical treatment measures, the rise of the epidemic situation in China has been contained to a certain extent, and the epidemic situation has eased in most provinces, but the incidence abroad is on the rise. With increased understanding of the clinical manifestations and pathology of the disease, and the accumulation of experience in diagnosis and treatment, in order to further strengthen the early diagnosis and early treatment of the disease, improve the cure rate, reduce the mortality rate, avoid nosocomial infection as much as possible and pay attention to the spread caused by the imported cases from overseas, we revised the Diagnosis and Treatment Protocol for Novel Coronavirus Pneumonia (Trial Version 6) to Diagnosis and Treatment Protocol for Novel Coronavirus Pneumonia (Trial Version 7).

\section{Etiological Characteristics}

The novel coronaviruses belong to the $\beta$ genus. They have envelopes, and the particles are round or oval, often polymorphic, with diameter being 60 to $140 \mathrm{~nm}$. Their genetic characteristics are significantly different from SARS-CoV and MERS-CoV. Current research shows that they share more than $85 \%$ homology with bat SARS-like coronaviruses (bat-SL-CoVZC45). When isolated and cultured in vitro, the 2019-nCoV can be found in human respiratory epithelial cells in about 96 hours, however it takes about 6 days for the virus to be found if isolated and cultured in Vero E6 and Huh-7 cell lines.

Most of the know-how about the physical and chemical properties of coronavirus comes from the research on SARS-CoV and MERS-CoV. The virus is sensitive to ultraviolet and heat. Exposure to $56^{\circ} \mathrm{C}$ for 30 minutes and lipid solvents such as ether, $75 \%$ ethanol, 
chlorine-containing disinfectant, peracetic acid, and chloroform can effectively inactivate the virus. Chlorhexidine has not been effective in inactivating the virus.

\section{Epidemiological Characteristics}

\section{Source of infection}

Now, the patients infected by the novel coronavirus are the main source of infection; asymptomatic infected people can also be an infectious source.

\section{Route of transmission}

Transmission of the virus happens mainly through respiratory droplets and close contact. There is the possibility of aerosol transmission in a relatively closed environment for a long-time exposure to high concentrations of aerosol. As the novel coronavirus can be isolated in feces and urine, attention should be paid to feces or urine contaminated environmental that leads to aerosol or contact transmission.

3. Susceptible groups

People are generally susceptible.

\section{Pathological changes}

Pathological findings from limited autopsies and biopsy studies are summarized below:

1. Lungs

Solid changes of varying degrees are present in the lungs.

Alveolar damage involves fibromyxoid exudation and hyaline membrane formation. The exudates are composed of monocytes and macrophages, with plenty of multinucleated syncytial cells. Type II alveolar epithelial cells are markedly hyperplastic, some of which are desquamated. Viral inclusions are observed in type II alveolar epithelial cells and macrophages. Alveolar interstitium is marked with vascular congestion and edema, infiltration of monocytes and lymphocytes, and vascular hyaline thrombi. The lungs are laden with hemorrhagic and necrotic foci, along with evidence of hemorrhagic infarction. Organization of alveolar exudates and interstitial fibrosis are partly present.

The bronchi are filled with desquamated epithelial cells, mucus and mucus plugs. Hyperventilated alveoli, interrupted alveolar interstitium and cystic formation are occasionally seen.

On electron microscopy, cytoplasmic NCP virions are observed in the bronchial epithelium and type II alveolar epithelium. NCP virus antigen positivity in some alveolar epithelia and macrophages are revealed through immunohistochemistry staining, which are positive for 
NCP virus nucleic acid via RT-PCR.

2. Spleen, hilar lymph nodes and bone marrow

The spleen is evidently shrunk. Lymphocytopenia and focal hemorrhage and necrosis are present. Macrophagocyte proliferation and phagocytosis are noted in the spleen. Lymph nodes are found with sparse lymphocytes and occasional necrosis. CD4+ and CD8+ T cells are present in reduced quantity in the spleen and lymph nodes, revealed by immunohistochemistry staining. Pancytopenia is identified in bone marrow.

3. Heart and blood vessels

Degenerated or necrosed myocardial cells are present, along with mild infiltration of monocytes, lymphocytes and/or neutrophils in the cardiac interstitium. Endothelial desquamation, endovasculitis and thrombi are seen in some blood vessels.

4. Liver and gall bladder

Appearing enlarged and dark-red, the liver is found degenerated with focal necrosis infiltrated with neutrophils. The liver sinusoids are found hyperemic. The portal areas are infiltrated with lymphocytes and monocytes and dotted with microthrombi. The gall bladder is prominently filled.

5. Kidneys

The kidneys are noted with protein exudation in the Bowman's capsule around glomeruli, degeneration and desquamation of the epithelial cells of renal tubules, and hyaline casts. Microthrombi and fibrotic foci are found in the kidney interstitium.

6. Other organs

Cerebral hyperemia and edema are present, with degeneration of some neurons. Necrosis foci are noted in the adrenal glands. Degeneration, necrosis and desquamation of epithelium mucosae at varying degrees are present in the esophageal, stomach and intestine.

\section{Clinical Characteristics}

\section{Clinical manifestations}

Based on the current epidemiological investigation, the incubation period is one to 14 days, mostly three to seven days.

Main manifestations include fever, fatigue and dry cough. Nasal congestion, runny nose, sore throat, myalgia and diarrhea are found in a few cases. Severe cases mostly developed dyspnea and/or hypoxemia after one week. In severe cases, patients progress rapidly to acute respiratory distress syndrome, septic shock, metabolic acidosis that is difficult to correct, coagulopathy, multiple organ failure and others. It is worth noting that for severe 
and critically ill patients, their fever could be moderate to low, or even barely noticeable. Some children and neonatal cases may have atypical symptoms, manifested as gastrointestinal symptoms such as vomiting and diarrhea, or only manifested as low spirits and shortness of breath.

The patients with mild symptoms did not develop pneumonia but only low fever and mild fatigue.

From current situations, most patients have good prognosis and a small number of patients are critically ill. The prognosis for the elderly and patients with chronic underlying diseases is poor. The clinical course of pregnant women with NCP is similar to that of patients of the same age. Symptoms in children are relatively mild.

\section{Laboratory tests}

\section{General findings}

In the early stages of the disease, peripheral WBC count is normal or decreased and the lymphocyte count decreases. Some patients see an increase in liver enzymes, lactate dehydrogenase (LDH), muscle enzymes and myoglobin. Elevated troponin is seen in some critically ill patients while most patients have elevated C-reactive protein and erythrocyte sedimentation rate and normal procalcitonin. In severe cases, D-dimer increases and peripheral blood lymphocytes progressively decrease. Severe and critically ill patients often have elevated inflammatory factors.

Pathogenic and serological findings

(1) Pathogenic findings: Novel coronavirus nucleic acid can be detected in nasopharyngeal swabs, sputum, lower respiratory tract secretions, blood, feces and other specimens using RT-PCR and/or NGS methods. It is more accurate if specimens from lower respiratory tract (sputum or air tract extraction) are tested. The specimens should be submitted for testing as soon as possible after collection.

(2) Serological findings: NCP virus specific IgM becomes detectable around 3-5 days after onset; IgG reaches a titration of at least 4-fold increase during convalescence compared with the acute phase.

\section{Chest imaging}

In the early stage, imaging shows multiple small patchy shadows and interstitial changes, apparent in the outer lateral zone of lungs. As the disease progresses, imaging then shows multiple ground glass opacities and infiltration in both lungs. In severe cases, pulmonary 
consolidation may occur while pleural effusion is rare.

\section{Case Definitions}

\section{Suspect cases}

Considering both the following epidemiological history and clinical manifestations:

\subsection{Epidemiological history}

1.1.1 History of travel to or residence in Wuhan and its surrounding areas, or in other communities where cases have been reported within 14 days prior to the onset of the disease;

1.1.2 In contact with novel coronavirus infected people (with positive results for the nucleic acid test) within 14 days prior to the onset of the disease;

1.1.3 In contact with patients who have fever or respiratory symptoms from Wuhan and its surrounding area, or from communities where confirmed cases have been reported within 14 days before the onset of the disease; or

1.1.4 Clustered cases ( 2 or more cases with fever and/or respiratory symptoms in a small area such families, offices, schools etc within 2 weeks).

\subsection{Clinical manifestations}

\subsubsection{Fever and/or respiratory symptoms;}

\subsubsection{The aforementioned imaging characteristics of NCP;}

1.2.3 Normal or decreased WBC count, normal or decreased lymphocyte count in the early stage of onset.

A suspect case has any of the epidemiological history plus any two clinical manifestations or all three clinical manifestations if there is no clear epidemiological history.

\section{Confirmed cases}

Suspect cases with one of the following etiological or serological evidences:

2.1 Real-time fluorescent RT-PCR indicates positive for new coronavirus nucleic acid;

2.2 Viral gene sequence is highly homologous to known new coronaviruses.

2.3 NCP virus specific Ig M and IgG are detectable in serum; NCP virus specific IgG is detectable or reaches a titration of at least 4-fold increase during convalescence compared with the acute phase.

\section{Clinical Classification}


1. Mild cases

The clinical symptoms were mild, and there was no sign of pneumonia on imaging.

2. Moderate cases

Showing fever and respiratory symptoms with radiological findings of pneumonia.

3. Severe cases

Adult cases meeting any of the following criteria:

(1) Respiratory distress ( $\geqq 30$ breaths/ min);

(2) Oxygen saturation $\leqslant 93 \%$ at rest;

(3) Arterial partial pressure of oxygen $\left(\mathrm{PaO}_{2}\right) /$ fraction of inspired oxygen $\left(\mathrm{FiO}_{2}\right) \leqq$ $300 \mathrm{mmHg}(\mathrm{l} \mathrm{mmHg}=0.133 \mathrm{kPa})$.

In high-altitude areas (at an altitude of over 1,000 meters above the sea level), $\mathrm{PaO}_{2 /} \mathrm{FiO}_{2}$ shall be corrected by the following formula:

$\mathrm{PaO}_{2 /} \mathrm{FiO}_{2}$ x [Atmospheric pressure (mmHg)/760]

Cases with chest imaging that showed obvious lesion progression within $24-48$ hours $>50 \%$ shall be managed as severe cases.

Child cases meeting any of the following criteria:

(1) Tachypnea ( $R R \geq 60$ breaths/min for infants aged below 2 months; $R R \geq 50$ BPM for infants aged 2-12 months; RR $\geq 40 \mathrm{BPM}$ for children aged $1-5$ years, and $\mathrm{RR} \geq 30 \mathrm{BPM}$ for children above 5 years old) independent of fever and crying;

(2) Oxygen saturation $\leq 92 \%$ on finger pulse oximeter taken at rest;

(3) Labored breathing (moaning, nasal fluttering, and infrasternal, supraclavicular and intercostal retraction), cyanosis, and intermittent apnea;

(4) Lethargy and convulsion;

(5) Difficulty feeding and signs of dehydration.

4. Critical cases

Cases meeting any of the following criteria:

4.1 Respiratory failure and requiring mechanical ventilation;

4.2 Shock;

4.3 With other organ failure that requires ICU care.

\section{Clinical early warning indicators of severe and critical cases}

1. Adults. 
1.1 The peripheral blood lymphocytes decrease progressively;

1.2 Peripheral blood inflammatory factors, such as IL-6 and C-reactive proteins, increase progressively;

1.3 Lactate increases progressively;

1.4 Lung lesions develop rapidly in a short period of time.

2. Children.

2.1 Respiratory rate increased;

2.2 Poor mental reaction and drowsiness;

2.3 Lactate increases progressively;

2.4 Imaging shows infiltration on both sides or multiple lobes, pleural effusion or rapid progress of lesions in a short period of time;

2.5 Infants under the age of 3 months who have either underlying diseases (congenital heart disease, bronchopulmonary dysplasia, respiratory tract deformity, abnormal hemoglobin, and severe malnutrition, etc.) or immune deficiency or hypofunction (long-term use of immunosuppressants).

\section{Differential Diagnosis}

1. The mild manifestations of NCP need to be distinguished from upper respiratory tract infections caused by other viruses.

2. NCP is mainly distinguished from other known viral pneumonia and mycoplasma pneumoniae infections such as influenza virus, adenovirus and respiratory syncytial virus. Especially for suspect cases, methods such as rapid antigen detection and multiplex PCR nucleic acid detection should be adopted as much as possible for detection of common respiratory pathogens.

3. It should also be distinguished from non-infectious diseases such as vasculitis, dermatomyositis and organizing pneumonia.

\section{Case Finding and Reporting}

Health professionals in medical institutions of all types and at all levels, upon discovering suspect cases that meet the definition, should immediately put them in single room for isolation and treatment. If the cases are still considered as suspected after consultation made by hospital experts or attending physicians, it should be reported directly online within 2 hours; samples should be collected for new coronavirus nucleic acid testing and 
suspect cases should be safely transferred to the designated hospitals immediately. People who have been in close contact with patients who have been confirmed of new coronavirus infection are advised to perform new coronavirus pathogenic testing in a timely manner, even though common respiratory pathogens are tested positive.

If two nucleic acid tests, taken at least 24-hour apart, of a NCP suspect case are negative, and the NCP virus specific IgM and IgG are negative after 7 days from onset, the suspect diagnosis can be ruled out.

\section{Treatment}

1. Treatment venue determined by the severity of the disease

1.1 Suspected and confirmed cases should be isolated and treated at designated hospitals with effective isolation, protection and prevention conditions in place. A suspect case should be treated in isolation in a single room. Confirmed cases can be treated in the same room.

1.2 Critical cases should be admitted to ICU as soon as possible.

\section{General treatment}

2.1 Letting patients rest in bed and strengthening support therapy; ensuring sufficient caloric intake for patients; monitoring their water and electrolyte balance to maintain internal environment stability; closely monitoring vital signs and oxygen saturation.

2.2 According to patients' conditions, monitoring blood routine result, urine routine result, c-reactive protein (CRP), biochemical indicators (liver enzyme, myocardial enzyme, renal function etc.), coagulation function, arterial blood gas analysis, chest imaging and cytokines detection if necessary.

2.3 Timely providing effective oxygen therapy, including nasal catheter and mask oxygenation and nasal high-flow oxygen therapy. If possible, inhalation of mixed hydrogen and oxygen $\left(\mathrm{H}_{2} / \mathrm{O}_{2}: 66.6 \% / 33.3 \%\right)$ can be applied.

2.4 Antiviral therapy: Hospitals can try Alpha-interferon (5 million U or equivalent dose each time for adults, adding $2 \mathrm{ml}$ of sterilized water, atomization inhalation twice daily), lopinavir/ritonavir (200 mg/50mg per pill for adults, two pills each time, twice daily, no longer than 10 days), Ribavirin (suggested to be used jointly with interferon or lopinavir/ritonavir, $500 \mathrm{mg}$ each time for adults, twice or three times of intravenous injection daily, no longer than 10 days), chloroquine phosphate (500 mg bid for 7 days for adults aged 18-65 with body weight over 50 kg; 500 mg bid for Days $1 \& 2$ and 500 mg qd 
for Days 3-7 for adults with body weight below $50 \mathrm{~kg}$ ), Arbidol (200 mg tid for adults, no longer than 10 days). Be aware of the adverse reactions, contraindications (for example, chloroquine cannot be used for patients with heart diseases) and interactions of the abovementioned drugs. Further evaluate the efficacy of those drugs currently being used. Using three or more antiviral drugs at the same time is not recommend; if an intolerable toxic side effect occurs, the respective drug should be discontinued. For the treatment of pregnant women, issues such as the number of gestational weeks, choice of drugs having the least impact on the fetus, as well as whether pregnancy being terminated before treatment should be considered with patients being informed of these considerations.

2.5 Antibiotic drug treatment: Blind or inappropriate use of antibiotic drugs should be avoided, especially in combination with broad-spectrum antibiotics.

\section{Treatment of severe and critical cases}

3.1 Treatment principle: On the basis of symptomatic treatment, complications should be proactively prevented, underlying diseases should be treated, secondary infections also be prevented, and organ function support should be provided timely.

\subsection{Respiratory support:}

3.2.1 Oxygen therapy: Patients with severe symptoms should receive nasal cannulas or masks for oxygen inhalation and timely assessment of respiratory distress and/or hypoxemia should be performed.

3.2.2 High-flow nasal-catheter oxygenation or noninvasive mechanical ventilation: When respiratory distress and/or hypoxemia of the patient cannot be alleviated after receiving standard oxygen therapy, high-flow nasal cannula oxygen therapy or non-invasive ventilation can be considered. If conditions do not improve or even get worse within a short time (1-2 hours), tracheal intubation and invasive mechanical ventilation should be used in a timely manner.

3.2.3 Invasive mechanical ventilation: Lung protective ventilation strategy, namely low tidal volume (6-8ml/kg of ideal body weight) and low level of airway platform pressure $\left(<30 \mathrm{cmH}_{2} \mathrm{O}\right)$ should be used to perform mechanical ventilation to reduce ventilator-related lung injury. While the airway platform pressure maintained $\leqslant 30 \mathrm{cmH}_{2} \mathrm{O}$, high PEEP can be used to keep the airway warm and moist; avoid long sedation and wake the patient early for lung rehabilitation. There are many cases of human-machine asynchronization, therefore sedation and muscle relaxants should be used in a timely manner. Use closed sputum suction according to the airway secretion, if necessary, administer appropriate 
treatment based on bronchoscopy findings.

3.2.4 Rescue therapy: Pulmonary re-tensioning is recommended for patients with severe ARDS. With sufficient human resources, prone position ventilation should be performed for more than 12 hours per day. If the outcome of prone position ventilation is poor, extracorporeal membrane oxygenation (ECMO) should be considered as soon as possible. Indications include: (1)When Fi02 $>90 \%$, the oxygenation index is less than $80 \mathrm{mmHg}$ for more than 3-4 hours; (2)For patients with only respiratory failure when the airway platform pressure $\geq 35 \mathrm{cmH} 2 \mathrm{O}, \mathrm{VV}$-ECMO mode is preferred; if circulatory support is needed, VAECMO mode should be used. When underlying diseases are under control and the cardiopulmonary function shows signs of recovery, withdrawal of ECMO can be tried.

3.3 Circulatory support: On the basis of adequate fluid resuscitation, efforts should be made to improve microcirculation, use vasoactive drugs, closely monitor changes in blood pressure, heart rate and urine volume as well as lactate and base excess in arterial blood gas analysis. If necessary, use non-invasive or invasive hemodynamic monitor such as Doppler ultrasound, echocardiography, invasive blood pressure or continuous cardiac output (PiCCO) monitoring. In the process of treatment, pay attention to the liquid balance strategy to avoid excessive or insufficient fluid intake.

If the heart rate suddenly increases more than $20 \%$ of the basic value or the decrease of blood pressure is more than $20 \%$ of the basic value with manifestations of poor skin perfusion and decreased urine volume, make sure to closely observe whether the patient has septic shock, gastrointestinal hemorrhage or heart failure.

3.4 Renal failure and renal replacement therapy: Active efforts should be made to look for causes for renal function damage in critical cases such as low perfusion and drugs. For the treatment of patients with renal failure, focus should be on the balance of body fluid, acid and base and electrolyte balance, as well as on nutrition support including nitrogen balance and the supplementation of energies and trace elements. For critical cases, continuous renal replacement therapy (CRRT) can be used. The indications include: (1) hyperkalemia; (2) acidosis; (3) pulmonary edema or water overload; (4) fluid management in multiple organ dysfunction.

3.5 Convalescent plasma treatment: It is suitable for patients with rapid disease progression, severe and critically ill patients. Usage and dosage should refer to Protocol of Clinical Treatment with Convalescent Plasma for NCP Patients ( $2^{\text {nd }}$ trial version).

3.6 Blood purification treatment: Blood purification system including plasma exchange, 
absorption, perfusion and blood/plasma filtration can remove inflammatory factors and block "cytokine storm", so as to reduce the damage of inflammatory reactions to the body. It can be used for the treatment of severe and critical cases in the early and middle stages of cytokine storm.

3.7 Immunotherapy: For patients with extensive lung lesions and severe cases who also show an increased level of IL-6 in laboratory testing, Tocilizumab can be used for treatment. The initial dose is $4-8 \mathrm{mg} / \mathrm{kg}$ with the recommended dose of $400 \mathrm{mg}$ diluted with $0.9 \%$ normal saline to $100 \mathrm{ml}$. The infusion time should be more than 1 hour. If the initial medication is not effective, one extra administration can be given after 12 hours (same dose as before). No more than two administrations should be given with the maximum single dose no more than $800 \mathrm{mg}$. Watch out for allergic reactions. Administration is forbidden for people with active infections such as tuberculosis.

3.8 Other therapeutic measures

For patients with progressive deterioration of oxygenation indicators, rapid progress in imaging and excessive activation of the body's inflammatory response, glucocorticoids can be used in a short period of time (three to five days). It is recommended that dose should not exceed the equivalent of methylprednisolone $1-2 \mathrm{mg} / \mathrm{kg} /$ day. Note that a larger dose of glucocorticoid will delay the removal of coronavirus due to immunosuppressive effects. Xuebijing 100ml/time can be administered intravenously twice a day. Intestinal microecological regulators can be used to maintain intestinal microecological balance and prevent secondary bacterial infections.

Child severe and critical cases can be given intravenous infusion of $\gamma$-globulin.

For pregnant severe and critical cases, pregnancy should be terminated preferably with csection.

Patients often suffer from anxiety and fear and they should be supported by psychological counseling.

4. Traditional Chinese Medicine treatment

This disease belongs to the category of plague in traditional Chinese medicine (TCM), caused by the epidemic pathogenic factors. According to the different local climate characteristic and individual state of illness and physical conditions, the following treatment Protocol may vary. The use of over-pharmacopoeia doses should be directed by a physician.

\subsection{During medical observation}


Clinical manifestation 1: fatigue and gastrointestinal discomfort

Recommended Chinese patent medicine: Huoxiang Zhengqi capsules (pills, liquid, or oral solution)

Clinical manifestation 2: fatigue and fever

Recommended Chinese patent medicine: Jinhua Qinggan granules, Lianhua Qingwen capsules (granules), Shufeng Jiedu capsules (granules), Fangfeng Tongsheng pills (granules)

\subsection{During clinical treatment (confirmed cases)}

\subsubsection{Lung cleansing \& detoxifying decoction}

Scope of application: It is suitable for light, moderate and severe patients, and can be used reasonably in combination with the actual situation of patients in the treatment of critically ill patients.

Recommended prescription: Ephedra 9g, Zhigancao 6g, Almond 9g, Gypsum 15-30g (fried first), Guizhi 9g, Zixie 9g, Zhuling 9g, Baizhu 9g, Zhiling 15g, Bupleurum 16g, Scutellaria baicalensis 6g, and Pinellia 9g, Ginger 9g, aster 9g, winter flower 9g, shoot dry 9g, asarum 6g, yam 12g, coriander fruit 6g, tangerine peel 6g, aquilegia 9g. Suggested use: Traditional Chinese medicine decoction pieces for decocting in water. One dose per day, twice in the morning and evening (forty minutes after a meal), take with warm water, and three doses a course.

If conditions permit, the patient can take half a bowl of rice soup each time after taking the medicine, and can take up to one bowl if the patient has a dry tongue and is deficient in bodily fluids. (Note: If the patient does not have a fever, the amount of gypsum should be little. If having a fever or strong heat, the amount of gypsum can be increased). If the symptoms improve but do not fully recover, then take the second course of treatment. If the patient has special conditions or other underlying diseases, the prescription of the second course of treatment can be modified based on the actual situation and the medicine should be discontinued when the symptoms disappear.

Source of prescription: Notice on Recommending the Use of 'Lung cleansing \& detoxifying decoction' in Treatment of NCP by Integrated Traditional Chinese and Western Medicine by the Office of the State Administration of Traditional Chinese Medicine \& the General Office of the National Health Commission. (2022 No.22)

\subsubsection{Mild cases}




\subsubsection{Cold dampness and stagnation lung syndrome}

Clinical manifestations: fever, fatigue, sore body, cough, expectoration, chest tightness, suffocation, loss of appetite, nausea, vomiting, sticky stools. Tongue has thin fat tooth mark or is faint red, and the coating is white thick rot or white greasy and the pulse is moisten or slippery.

Recommended prescription: Raw ephedra 6g, raw gypsum 15g, almond 9g, loquat 15g, gardenia 15g, Guanzhong 9g, Dilong 15g, Xu Changqing 15g, Huoxiang 15g, Peilan 9g, Cangzhu 15g, Yunling 45g, Atractylodes 30g, Jiao Sanxian 9g each , Magnolia officinalis $15 \mathrm{~g}$, betel coconut $9 \mathrm{~g}$, yarrow fruit $9 \mathrm{~g}$, ginger $15 \mathrm{~g}$.

Suggested use: one dose daily, boiled with $600 \mathrm{ml}$ water, take it three times at morning, noon and evening before meal.

\subsubsection{Dampness and heat-accumulation lung syndrome}

Clinical manifestations: low or no fever, slight chills, fatigue, heavy head and body, muscle soreness, dry cough, low phlegm, sore throat, dry mouth, do not want to drink more, or accompanied by chest tightness, no sweat or sweating, Or vomiting and loss of appetite, diarrhea or sticky stool. The tongue is reddish, and the coating is white, thick and greasy or thin yellow, and the pulse is slippery or sloppy.

Recommended prescription: Betel nut 10g, apple 10g, Magnolia 10g, Zhimu 10g, scutellaria baicalensis $10 \mathrm{~g}$, Bupleurum $10 \mathrm{~g}$, red peony $10 \mathrm{~g}$, forsythia $15 \mathrm{~g}$, artemisia annua $10 \mathrm{~g}$ (decocted later), $10 \mathrm{~g}$ of green leaves, $10 \mathrm{~g}$ of green leaves, $5 \mathrm{~g}$ of raw licorice.

Suggested use: one dose daily, boiled with 400ml water, take it twice at morning and evening.

\subsubsection{Moderate cases}

\subsubsection{Dampness and stagnation lung syndrome}

Clinical manifestations: fever, low cough and sputum, or yellow sputum, suffocation, shortness of breath, bloating, and constipation. The tongue is dark red and fat; the coating is greasy or yellow and the pulse is slippery or stringy.

Recommended prescription: raw ephedra 6g, bitter almond 15g, raw gypsum 30g, raw coix seed $30 \mathrm{~g}$, grass root $10 \mathrm{~g}$, patchouli 15g, artemisia annua 12g, Polygonum cuspidatum $20 \mathrm{~g}$, verbena $30 \mathrm{~g}$, dried reed root $30 \mathrm{~g}$, gardenia $15 \mathrm{~g} 15 \mathrm{~g}$ of orange red, $10 \mathrm{~g}$ of raw licorice. Suggested use: one dose daily, boiled with $400 \mathrm{ml}$ water, take it twice at morning and evening. 


\subsubsection{Cold dampness lung syndrome}

Clinical manifestations: low fever, low body temperature, or no heat, dry cough, low sputum, fatigue, chest tightness, nausea, or nausea. The tongue is pale or red, and the coating is white or greasy, and the veins are pulsating.

Recommended prescription: Atractylodes lancea 15g, Chenpi 10g, Magnolia 10g, Aquilegia 10g, grass fruit 6g, raw ephedra 6g, Zhihuo 10g, ginger $10 \mathrm{~g}$, betel nut $10 \mathrm{~g}$.

Suggested use: one dose daily, boiled with $400 \mathrm{ml}$ water, take it twice at morning and evening.

\subsubsection{Severe cases}

\subsubsection{Plague poison and lung-closing syndrome}

Clinical manifestations: fever, flushing, cough, yellowish phlegm, or blood in sputum, wheezing, shortness of breath, tiredness, fatigue, dryness and stickiness, nausea, food loss, poor stool, and short urination. Red tongue, yellow greasy coating, slippery pulses.

Recommended prescription: Raw ephedra 6g, almond 9g, raw gypsum 15g, licorice 3g, fragrant fragrant 10g (back), Magnolia 10g, atractylodes 15g, grass fruit 10g, pinellia 9g, Poria $15 \mathrm{~g}$, raw rhubarb $5 \mathrm{~g}$ (back) $10 \mathrm{~g}$, gardenia $10 \mathrm{~g}$, red peony $10 \mathrm{~g}$.

Suggested use: one or two doses daily, boiled with 100-200ml water, take it 2-4 times, oral or nasal feeding.

\subsubsection{Syndrome of flaring heat in qifen and yingfen}

Clinical manifestations: Hot fever, thirst, shortness of breath, shortness of breath, blurred vision, or spotted rash, or vomiting blood, bleeding, or convulsions in the limbs. Tongue ridges have few or no moss, and the pulse sinks finely, or floats large and counts.

Recommended prescription: 30-60g gypsum (fried first), 30g of Zhimu, 30-60g of raw land, $30 \mathrm{~g}$ of buffalo horn (fried first), 30g of red sage, 30g of black ginseng, $15 \mathrm{~g}$ of forsythia, $15 \mathrm{~g}$ of paeonia, $6 \mathrm{~g}$ of peony $12 \mathrm{~g}$, gardenia $15 \mathrm{~g}$, raw licorice $6 \mathrm{~g}$.

Suggested use: 1 dose per day, decoction, first decoct gypsum and buffalo horn, then apply other pieces, $100 \mathrm{ml}-200 \mathrm{ml}$ each time, 2-4 times a day, orally or nasally.

Recommended Chinese patent medicines: Xiyanping injection, Xuebijing injection, Reduning injection, Tanreqing injection, Xingnaojing injection. Drugs with similar efficacy can be selected according to individual conditions, or can be used in combination according to clinical symptoms. Traditional Chinese medicine injection can be used in 
combination with traditional Chinese medicine decoction.

\subsubsection{Critical cases (syndrome of inner blocking causing collapse)}

Clinical manifestations: dyspnea, dyspnea, asthma or need mechanical ventilation, fainting, irritability, cold sweating, dark purple tongue, thick or dry moss, large floating roots.

Recommended prescription: 15g of ginseng, $10 \mathrm{~g}$ of Heishun tablets (decoct first), 15g of dogwood, delivered with Suhexiang Pill or Angong Niuhuang Pill.

For patients on mechanical ventilation with abdominal distention or constipation: 5-10g of Dahuang. For patients with human-machine asynchronization: 5-10g of Dahuang and 5$10 \mathrm{~g}$ of Mangxiao while administering sedatives and muscle relaxants.

Recommended Chinese patent medicines: Xuebijing injection, Reduning injection, Tanreqing injection, Xingnaojing injection, Shenfu injection, Shengmai injection, Shenmai injection. Drugs with similar efficacy can be selected according to individual conditions, or can be used in combination according to clinical symptoms. Traditional Chinese medicine injection can be used in combination with traditional Chinese medicine decoction.

\section{Note: Recommended usage of Chinese medicine injections for severe and critical cases}

The use of traditional Chinese medicine injections follows the principle of starting from a small dose and gradually adjusting the dosage according to the instructions of the drug. The recommended usage is as follows:

Viral infection or combined mild bacterial infection: $0.9 \%$ sodium chloride injection $250 \mathrm{ml}$ plus Xiyanping injection $100 \mathrm{mg}$ bid, or $0.9 \%$ sodium chloride injection $250 \mathrm{ml}$ heated Duning injection $20 \mathrm{ml}$, or $0.9 \%$ sodium chloride injection $250 \mathrm{ml}$ plus Tanreqing injection $40 \mathrm{ml}$ bid.

High fever with disturbance of consciousness: $250 \mathrm{ml}$ of $0.9 \%$ sodium chloride injection and 20ml bid of Xingnaojing injection.

Systemic inflammatory response syndrome or/and multiple organ failure: $250 \mathrm{ml}$ of $0.9 \%$ sodium chloride injection and 100ml of Xuebijing injection.

Immunosuppression: $250 \mathrm{ml}$ of $0.9 \%$ sodium chloride injection and $100 \mathrm{ml}$ bid of Shenmai injection.

Shock: $250 \mathrm{ml}$ of $0.9 \%$ sodium chloride injection plus $100 \mathrm{ml}$ bid of Shenfu injection.

\subsubsection{Convalescent period}

4.2.6.1 Lung and spleen qi deficiency syndrome 
Clinical manifestations: shortness of breath, fatigue, fatigue, anorexia, nausea, fullness, weak stool, and uneasiness. The tongue is pale and greasy.

Recommended prescription: French Pinellia 9g, Chenpi 10g, Codonopsis 15g, Sunburn Astragalus 30g, Stir-fried Atractylodes 10g, Poria 15g, Huoxiang 10g, Amomum villosum $6 g$ (later), and Licorice $6 g$

Suggested use: 1 dose per day, boiled with 400ml of water, twice a day at morning and evening.

\subsubsection{Qi and Yin deficiency syndrome}

Clinical manifestations: Fatigue, shortness of breath, dry mouth, thirst, palpitations, sweating, poor appetite, low or no lever, dry cough and little sputum; dry tongue, fine or weak pulses.

Recommended prescription: North and south radix salviae $10 \mathrm{~g}, 15 \mathrm{~g}$ ophiopogonis, $6 \mathrm{~g}$ American ginseng, 6g schisandra, $6 \mathrm{~g}$ gypsum 15g, 10g light bamboo leaves, 10g mulberry leaves, $15 \mathrm{~g}$ reed root, $15 \mathrm{~g}$ salviae miltiorrhiza, $6 \mathrm{~g}$ raw liquorice.

Suggested use: 1 dose per day, boiled with $400 \mathrm{ml}$ of water, twice a day at morning and evening.

\section{Discharge criteria and after-discharge considerations}

1.Discharge criteria

1) Body temperature is back to normal for more than three days;

2) Respiratory symptoms improve obviously;

3) Pulmonary imaging shows obvious absorption of inflammation,

4) Nuclei acid tests negative twice consecutively on respiratory tract samples such as sputum and nasopharyngeal swabs (sampling interval being at least 24 hours).

Those who meet the above criteria can be discharged.

2. After-discharge considerations

2.1 The designated hospitals should contact the primary healthcare facilities where the patients live and share patients' medical record, to send the information of the discharged patients to the community committee and primary healthcare facility where the patients reside.

2.2. After discharge, it is recommended for patients to monitor their own health status in isolation for 14 days, wear a mask, live in well-ventilated single room if possible, reduce 
close contact with family members, separate dinning, practice hand hygiene and avoid going out.

2.3 It is recommended for the patients to return to the hospitals for follow-up and re-visit in two and four weeks after discharge.

\section{Patients Transportation Principles}

Patients should be transported in accordance with the Work Protocol for Transfer of the Novel Coronavirus Pneumonia Patients (Trial Version) issued by the National Health Commission.

\section{Nosocomial Infection Prevention and Control}

Measures to prevent and control nosocomial infection should be implemented in accordance with the requirements of the Technical Guidelines for the Prevention and Control of Infection by the Novel Coronavirus in Medical Institutions (First Edition) and the Guidelines on the Usage of Common Medical Protective Equipment against Novel Coronavirus Infection (Trial Version) formulated by the National Health Commission.

The General Office of National Health Commission

Office of State TCM Administration

Printed and distributed on March 3, 2020 